\title{
Conservation of imprinting of Neuronatin (Nnat) in rabbits
}

\author{
Feifei Duan ${ }^{\dagger}$, Xianju Chen ${ }^{\dagger}$, Lin Yuan, Yuning Song, Anfeng Wang, Qingyan Lv, Zhanjun Li ${ }^{*}$ and Liangxue Lai
}

\begin{abstract}
Although the expression and epigenetic status of imprinted genes have been extensively studied in a number of species, less is known about the genomic imprinting in rabbits. Neuronatin (Nnat) plays significant roles in the brain development and metabolic regulation and has been identified to be imprinted and paternally expressed in humans, mice and pigs; however, it has not yet been investigated in rabbits. In this study, we confirmed the expression of two isoforms of the rabbit Nnat (Nnat-a and Nnat- $\beta$ ) identified in Genbank and Ensembl by quantitative real-time PCR. In addition, we also determined the methylation profile of the $\mathrm{CpG}$ island in the promoter region of the rabbit Nnat using bisulfite sequencing PCR and combined bisulfite restriction analysis. Here, we provide the first evidence that Nnat has two transcripts in rabbit. Additionally, the CpG island located in the promoter region shows oocyte-specific methylation and may be the differentially methylated region of Nnat in rabbits.
\end{abstract}

Keywords: DMR, Imprinted gene, Nnat, Paternally expressed, Promoter, Rabbit

\section{Background}

The model organisms play an important role in the study of human diseases. Owing to the unique features of the lipoprotein metabolism and being sensitive to cholesterol diet, rabbit models have been widely used to study human atherosclerosis, and have also made a great contribution to the translational research (Fan et al. 2014). The genomic imprinting phenomenon is theorized to exist in all the placental mammals and plays an important role in the regulation of fetal growth, development, and postnatal behavior, however, little is known about imprinted genes in mammalian species apart from human and mouse (Zhang et al. 2012; Dindot et al. 2004). At present, 80 imprinted genes have been identified in humans, 125 in mice, but only 20 in cows, 16 in sheep and just one imprinted gene (Impact) was confirmed in rabbits (http://igc.otago.ac.nz/home.html) (Zhang et al. 2012).

The Nnat was discovered in the neonatal rat brain for the first time and was subsequently found to play a significant role in the neuronal development, it is maternally

\footnotetext{
*Correspondence: lizj_1998@jlu.edu.cn

${ }^{\dagger}$ Feifei Duan and Xianju Chen contributed equally to this work

College of Animal Science, Jilin University, 5333\#, Xi'an Road,

Changchun 130062, China
}

imprinted in humans, mice, cattle and pigs (Evans et al. 2001; Kagitani et al. 1997; Zaitoun and Khatib 2006; Cheng et al. 2007). The more recent data have also demonstrated that Nnat participates not only in neuronal growth, but also in pituitary development, glucose-mediated insulin secretion in the pancreas and keratinocyte differentiation in the skin (Joseph 2014; Chen et al. 2014). Additionally, the Nnat protein has been also found to be located in the aortic endothelium which may increase endothelial cell adhesion molecule expression mediated through phosphatidylinositol 3-kinase (PI 3-kinase)/

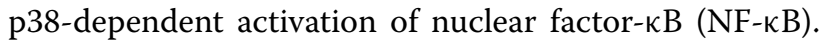
Therefore, Nnat is thought to be a candidate molecule that might be involved in inflammatory pathways associated with obesity and insulin resistance related to the endothelial dysfunction and/or the development of atherosclerosis (Mzhavia et al. 2008). All of the above findings suggest that Nnat plays a number of important roles in the mammalian development.

Although Nnat is known to be imprinted in many species, its imprinting status has not been determined in rabbits. In this study, we aimed to determine the expression levels of the rabbit Nnat in the brain, liver, kidney, eye and fetus by quantitative real-time PCR (qPCR). The bisulfite sequencing PCR (BSP) and combined bisulfite

\section{楚 Springer}

(c) 2015 Duan et al. This article is distributed under the terms of the Creative Commons Attribution 4.0 International License (http://creativecommons.org/licenses/by/4.0/), which permits unrestricted use, distribution, and reproduction in any medium, provided you give appropriate credit to the original author(s) and the source, provide a link to the Creative Commons license, and indicate if changes were made. 
restriction analysis (COBRA) were performed to further determine the imprinting status and differentially methylated region (DMR) of Nnat in different rabbit tissues including the brain, liver and germ cells.

\section{Results and discussion}

The Nnat, which is a highly conserved gene among different species including humans, cattle and pigs, is located within the 8.5-kb intron of the Blcap (Bladder CancerAssociated Protein) gene and contains three exons and has two alternatively spliced transcripts ( $\alpha$ and $\beta$ ) (Schulz et al. 2009; Cheng et al. 2007). To further understand the expression pattern of Nnat in rabbit, primers were designed according to the two alternatively spliced transcripts (Nnat- $\alpha$ and Nnat- $\beta$ ), which encode 81 and 54 amino acid proteins, respectively (Figure 1 ). RT-PCR and qPCR results demonstrated that both Nnat- $\alpha$ and Nnat $\beta$ are expressed in brain, eye and fetus of rabbit. Consistent with the EST-derived gene expression data, both the transcripts were highly expressed in brain but not expressed in liver and kidney (Figure 2). In contrast to rabbit, two transcripts of Nnat are widely expressed in most of tissues including liver and kidney in cattle (both fetal and adult) and in 2-month pig (Cheng et al. 2007; Zaitoun and Khatib 2006), which means that there are different Nnat expression profiles among different species. As Nnat is thought to play a number of important roles in the mammalian development, it would be very useful to identify the expression patterns and the imprinting status of the Nnat in rabbits in order to analyze the conservation of genomic imprinting among different species.

Previously we have shown that the Nnat promoter sequence is a highly conserved region and the expression of the Nnat is significantly associated with the methylation status of the CpG island located in the Nnat promoter sequence in the pigs (Chen et al. 2014). To identify whether the promoter was involved in the regulation of Nnat expression in rabbits, the DNA methylation profile of the promoter CpG island located $375 \mathrm{bp}$ upstream of the first ATG codon in exon 1 was determined in the sperm and MII oocytes (Figure 1). The data of BSP showed unmethylated and fully methylated promoter region of the Nnat in sperm (Figure 3a) and MII oocytes (Figure $3 \mathrm{~b}$ ), respectively. Similar findings were observed using the COBRA analysis in both samples (Figure 3f). Some epigenetic information must be differentially contributed from the two gametes, including known imprint control regions (ICRs) that maintain their allele-specific

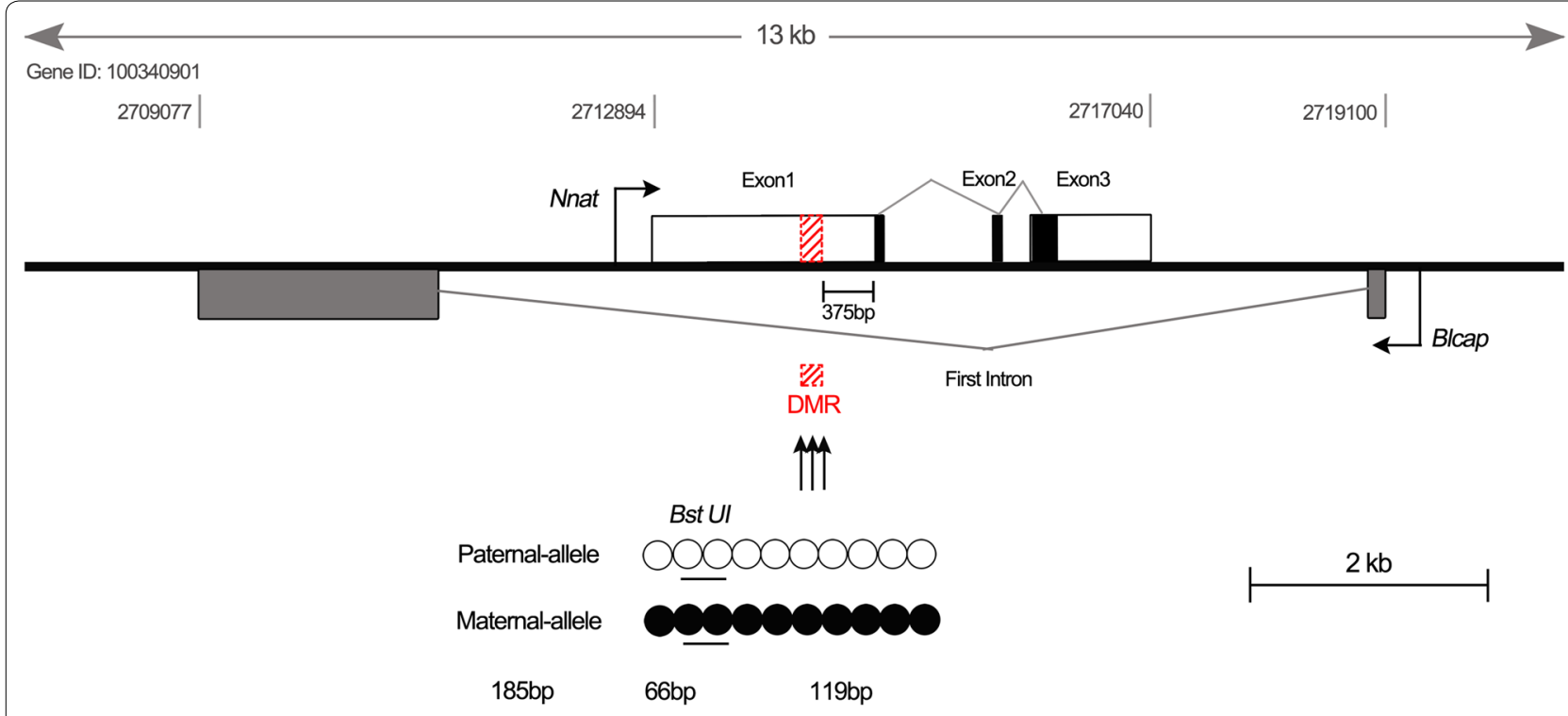

GgGATTITGGGTAGTAGAGAATTGGGTTATTATTTACGGAATATTGGGAAAGAGGGTT

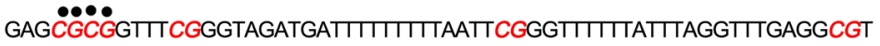
TIITTGTAGCGTTATTGGGTTGCGTTCGAATTITCGGTTAGGGTTAAGAAGAGGGGTATT

Figure 1 Structure of the Nnat locus in rabbits. The Nnat is located within the intron of Blcap and consists of three exons (white and black rectangular boxes) and two introns. The protein coding regions are indicated as black boxes. The gray boxes indicate Blcap. The black arrows indicate the transcription start sites of Nnat and Blcap. The box with red stripes within the promoter of Nnat represents the CpG island within the amplified region of BSP, which might be the DMR of the Nnat in rabbits. The circles indicate individual CpG dinucleotides within the BSP. Unmethylation (open) in the paternal-allele and methylation (closed) in the maternal-allele were presumed. Bisulfite-converted sequence of BSP products is shown with each $\mathrm{CpG}$ site marked in red; BstUI restriction sites (CGCG) are shown as black solid dots above the CpG sites and as bars below the circles. 


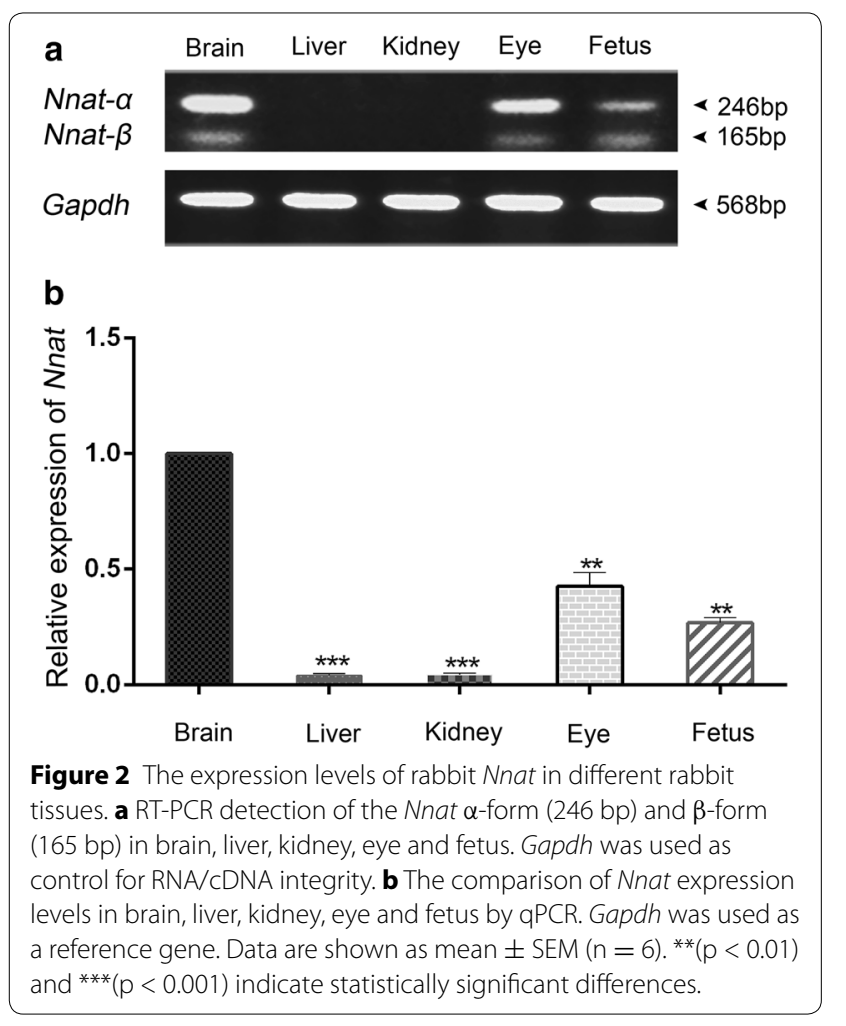

methylation pattern throughout embryogenesis (Smith et al. 2012). The gamete-specific methylation pattern of rabbit Nnat was consistent with our previous study which suggested that imprinting marks of Nnat were established via a sex-specific mechanism (Chen et al. 2014). Generally, the DNA methylation marks are established at zygote and primordial germ cells with a unique set of mechanisms regulating the DNA methylation erasure and re-establishment (Seisenberger et al. 2013). These findings suggested that the Nnat might be a paternally expressed gene in rabbits.

To further confirm the imprinting status of the rabbit Nnat, methylation profiles of the brain and liver tissues with or without the expression of Nnat were analysed using BSP and COBRA. The results showed that both methylated and unmethylated strands were obtained, in the identified CpG island in brain but hypermethylation was determined in liver (Figure $3 c-f$ ), indicating that the maternal allele might be methylated and the paternal allele unmethylated in a tissue-specific manner (Figure 1). The above findings suggest that Nnat is likely to be a paternally expressed gene, which is consistent with the previous reports on humans, mice, cattle and pigs (Evans et al. 2001; Kagitani et al. 1997; Zaitoun and Khatib 2006; Cheng et al.

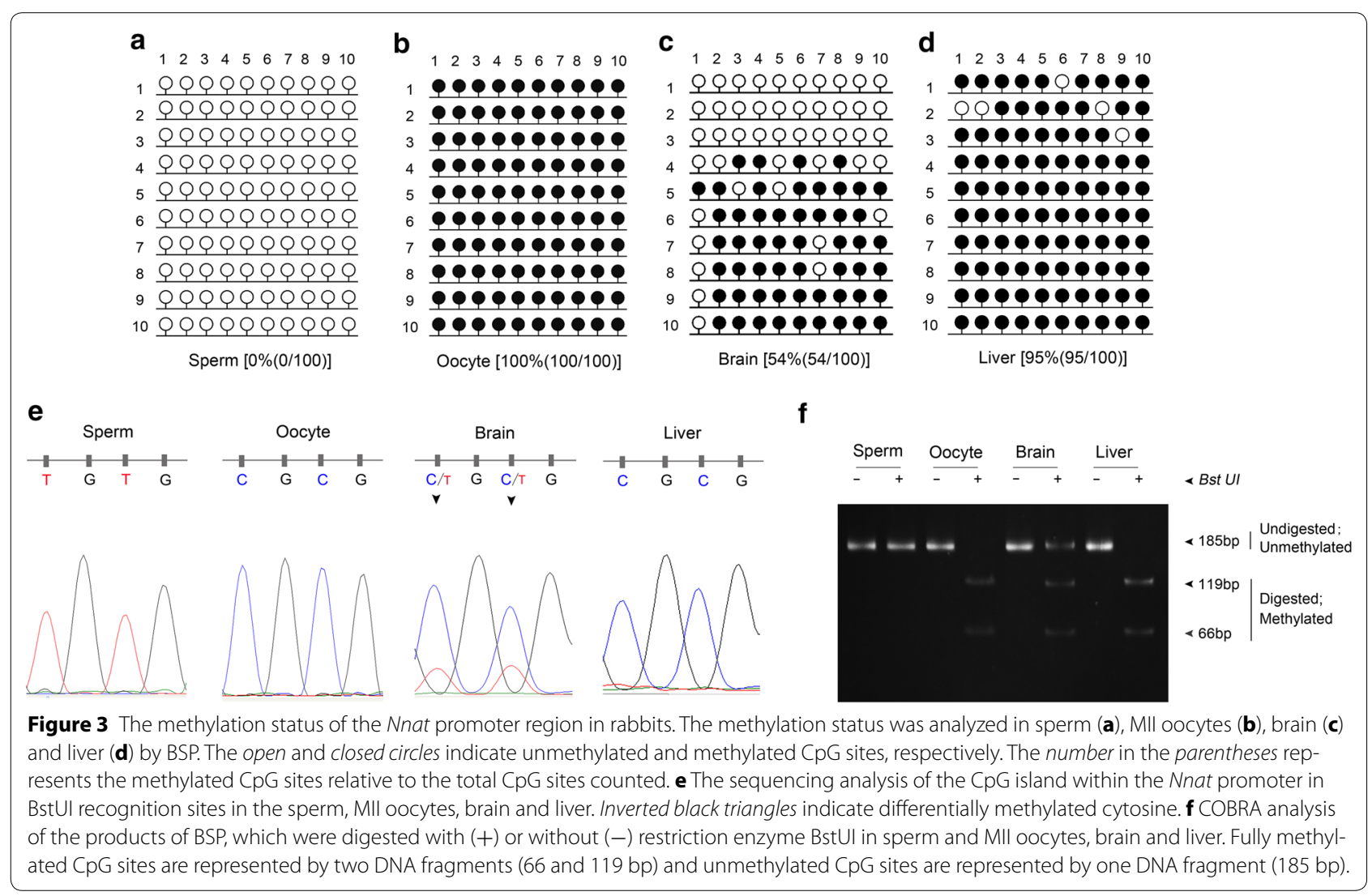


2007). Additionally, the partial methylation and hypermethylation of the Nnat promoter observed in rabbit brain and liver tissues, respectively, demonstrated that the Nnat gains methylation in non-expressing tissues. And the gamete-specific methylation pattern revealed the characteristics of an imprinted gene and identified the $\mathrm{CpG}$ promoter region being a putative DMR of the Nnat in rabbits.

\section{Conclusions}

Here, two transcripts of the Nnat in rabbit have been determined and the putative DMR located in the promoter sequence of Nnat in rabbits is identified for the first time. The gamete-specific methylation pattern of rabbit Nnat suggests that the Nnat is likely to be a paternally expressed gene in rabbits. In conclusion, we deduced that the conserved imprinting patterns among humans, mice, cattle and pigs suggest similar functions of Nnat in different mammalian species.

\section{Methods}

\section{Ethics statement}

The experimental procedures involving model organisms (rabbits) were performed in accordance with the specified guidelines on the animal care and the use of animals in research, which were approved by the Animal Care and Use Committee of the Jilin University, Changchun, China.

\section{Sample collection}

The brain, liver, kidney and eye were collected from 6-month old New Zealand White rabbits and normally fertilized fetuses were collected on Day 22 of gestation. All the samples were immediately stored in the liquid nitrogen till further use. MII oocytes were collected as described in a previous report (Tian et al. 2012). Briefly, sexually mature rabbits were superovulated by injecting six successive intramuscular doses of 60 IU of follicle-stimulating hormone (FSH; from Ningbo Second Hormone Factory) every $12 \mathrm{~h}$ and then intravenous injection with $100 \mathrm{IU}$ of human chorionic gonadotropin (hCG; from Ningbo Second Hormone Factory) after $12 \mathrm{~h}$ to induce ovulation. The cumulus-oocyte complexes (COCs) were flushed and collected from the oviducts at 13-14 h after the hCG injection. Mature oocytes were isolated from the COCs by $100 \mathrm{IU} / \mathrm{ml}$ hyaluronidase in M199. The pools of $100 \mathrm{MII}$ oocytes were frozen at $-80^{\circ} \mathrm{C}$ for further use in our study. Sperm were isolated using swim-up procedure. The purity and quality of the isolated germ cells were determined as described by an earlier report (Chen et al. 2014).

\section{Reverse transcription PCR (RT-PCR) and qPCR}

RT-PCR and qPCR were carried out as previously described (Chen et al. 2014). The primer sequences and
PCR amplification conditions are listed in Additional file 1: Table S1. The relative gene expression normalized to the Gapdh was determined by $2^{-\Delta \Delta C T}$ formula. All gene expression experiments were performed three times. The detection of gene expression was presented as mean $\pm \operatorname{SEM}(\mathrm{n}=6)$. The data were analyzed using student's $t$ tests with SPSS 16.0 software (SPSS Inc., Chicago, IL, USA) and $p<0.05$ was considered statistically significant.

\section{BSP and COBRA}

Bisulfite conversion and COBRA were performed according to previous study (Chen et al. 2014). Briefly, for bisulfite modification, genomic DNA from different tissues and germ cells was treated with the CpGenome ${ }^{\mathrm{Tm}}$ Turbo Bisulfite Modification Kit (Millipore) and EZ DNA Methylation-Direct TM Kit (Zymo Research), respectively. Primer sequences used for BSP are described in Additional file 1: Table S1. The PCR products were subjected to $\mathrm{T}$ vector cloning (positive clones, $\mathrm{n}=10$ ) and sequencing analysis, which showed heterozygosity at the $(\mathrm{C} / \mathrm{T})$ peak of Nnat. BSP products were also digested by a restriction enzyme BstUI (Thermo Scientific, MA, USA) for COBRA analysis. DNAMAN (LynnonBiosoft) and the online software tools MethOrimer and BiQ Analyzer were used for methylation analysis in this study.

\section{Additional files}

Additional file 1: Table S1. Primer sequences for RT-PCR, qRT-PCR and BSP.

\section{Abbreviations \\ Nnat: Neuronatin; PCR: polymerase chain reaction; qPCR: quantitative real-time PCR; BSP: bisulfite sequencing PCR; COBRA: combined bisulfite restriction analysis; PI 3-kinase: phosphatidylinositol 3-kinase; NF-кB: nuclear factor-кB; DMR: differentially methylated region; FSH: follicle-stimulating hormone; hCG: human chorionic gonadotropin; COCs: cumulus-oocyte complexes; RT-PCR: reverse transcription PCR; Blcap: Bladder Cancer-Associated Protein; ICRs: imprint control regions.}

\section{Authors' contribution}

FD, XC and ZL conceived, designed and performed the experiments described in this work and wrote the manuscript. LY and YS collected the samples. AW and QL performed the statistical analysis. LL provided guidance. All authors read and approved the final manuscript.

\section{Acknowledgements}

The authors express their gratitude to Xue Chen, Peiyan Hu at the Embryo Engineering Center for technical assistance. This work was financially supported by the National Natural Science Foundation of China (Grant No. 31201080 and 31272394). The Project was also funded through a grant from Special Financial Grant from the China Postdoctoral Science Foundation (2014T70295).

\section{Compliance with ethical guidelines}

\section{Competing interests}

The authors declare that they have no competing interests. 
Received: 19 November 2014 Accepted: 21 May 2015

Published online: 13 June 2015

\section{References}

Chen X, Wang T, Lv Q, Wang A, Ouyang H, Li Z (2014) DNA methylationmediated silencing of neuronatin (NNAT) in pig parthenogenetic fetuses. Gene 552(1):204-208

Cheng HC, Zhang FW, Deng CY, Jiang CD, Xiong YZ, Li FE et al (2007) NNAT and DIRAS3 genes are paternally expressed in pigs. Genet Sel Evol GSE 39(5):599-607

Dindot SV, Kent KC, Evers B, Loskutoff N, Womack J, Piedrahita JA (2004) Conservation of genomic imprinting at the XIST, IGF2, and GTL2 loci in the bovine. Mamm Genome 15(12):966-974

Evans HK, Wylie AA, Murphy SK, Jirtle RL (2001) The neuronatin gene resides in a "micro-imprinted" domain on human chromosome 20q11.2. Genomics 77:99-104

Fan J, Kitajima S, Watanabe T, Xu J, Zhang J, Liu E et al (2014) Rabbit models for the study of human atherosclerosis: From pathophysiological mechanisms to translational medicine. Pharmacol Ther 146:104-119

Joseph RM (2014) Neuronatin gene: imprinted and misfolded: Studies in Lafora disease, diabetes and cancer may implicate NNAT-aggregates as a common downstream participant in neuronal loss. Genomics 103(2-3):183-188
Kagitani F, Kuroiwa Y, Wakana S, Shiroishi T, Miyoshi N, Kobayashi S et al (1997) Peg5/neuronatin is an imprinted gene located on sub-distal chromosome 2 in the mouse. Nucleic Acids Res 25:3428-3432

Mzhavia N, Yu S, Ikeda S, Chu TT, Goldberg I, Dansky HM (2008) Neuronatin: a new inflammation gene expressed on the aortic endothelium of diabetic mice. Diabetes 57(10):2774-2783

Schulz R, McCole RB, Woodfine K, Wood AJ, Chahal M, Monk D et al (2009) Transcript- and tissue-specific imprinting of a tumour suppressor gene. Hum Mol Genet 18:118-127

Seisenberger S, Peat JR, Hore TA, Santos F, Dean W, Reik W (2013) Reprogramming DNA methylation in the mammalian life cycle: building and breaking epigenetic barriers. Philos Trans R Soc Lond B Biol Sci 368(1609):20110330

Smith ZD, Chan MM, Mikkelsen TS, Gu H, Gnirke A, Regev A et al (2012) A unique regulatory phase of DNA methylation in the early mammalian embryo. Nature 484(7394):339-344

Tian J, Song J, Li H, Yang D, Li X, Ouyang H et al (2012) Effect of donor cell type on nuclear remodelling in rabbit somatic cell nuclear transfer embryos. Reprod Domest Anim 47(4):544-552

Zaitoun I, Khatib H (2006) Assessment of genomic imprinting of SLC38A4, NNAT, NAP1L5, and H19 in cattle. BMC Genet 7:49

Zhang FW, Han ZB, Deng CY, He HJ, Wu Q (2012) Conservation of genomic imprinting at the NDN, MAGEL2 and MEST loci in pigs. Genes Genet Syst $87(1): 53-58$

\section{Submit your manuscript to a SpringerOpen ${ }^{\odot}$ journal and benefit from:}

- Convenient online submission

- Rigorous peer review

- Immediate publication on acceptance

- Open access: articles freely available online

- High visibility within the field

- Retaining the copyright to your article

Submit your next manuscript at $>$ springeropen.com 